\title{
THE PRINCIPLES OF TREATMENT OF POST-PARTUM HEMORRHAGE.
}

\author{
By WILLIAM HUNTER, M.D., M.C.O.G. \\ (Honorary Assistant Obstetriciai, Princess Mary Maternity Hospital, Newcastle-upon-Tyne.)
}

In the following paragraphs, the essentials of the treatment of atonic postpartum hæmorrhage will be summarized and briefly discussed.

This subject may be conveniently examined under the headings: "Preventive Treatment"; "Treatment of the Hæmorrhage"; and "Treatment of the Traumatic Anæmia," while the principles of treatment of the hæmorrhage may be epitomized in the terse sentences: "Empty the uterus-Stimulate the uterine muscle to contract-Control hæmorrhage while the uterus is regaining tone-Treat the collapse." These points will all be referred to in turn.

\section{Preventive Treatment.}

The uterus must not be manipulated during the third stage of labour. Kneading of the uterus before the placenta is completely separated commonly allows the retro-placental hæmatoma to escape prematurely, disturbs the normal mechanism of separation of the placenta so that the partially detached placenta or one of its cotyledons is not infrequently retained in utero and therefore predisposes to uterine hæmorrhage by interfering with muscular retraction ${ }^{(1,2,3)}$.

A distended bladder, uterine exhaustion following prolonged labour or antepartum hæmorrhage, rapid instrumental delivery in the presence of uterine inertia, or too free use of chloroform anæsthesia, may also be responsible for imperfect uterine retraction, and fundal massage or administration of oxytocic drugs, especially ergot, before the placenta is separated from the uterine wall may cause hour-glass contraction of the uterus, retention of the incompletely detached placenta and consequent imperfect retraction of the fundal myometrium.

If these predisposing causes were avoided, post-partum hæmorrhage would be less frequently encountered. Other possible predisposing factors, such as multiple pregnancies, fibromyomata and obesity, less frequently give rise to serious trouble, but, when this occurs, treatment is liable to be difficult.

\section{Treatment of the Hæmorrhage.}

Empty the Uterus. When uterine hæmorrhage not due to laceration of the genital tract follows delivery and does not cease after fundal massage per abdomen, the uterine contents should be evacuated without delay(4). A steady, continuous trickle of blood is no less important than a smart hæmorrhage, and may exsanguinate a patient in a comparatively short space of time.

If the placenta is still in the uterine cavity, it should be displaced by Crédés method, without or with general anæsthesia, by injection of normal saline solution into the umbilical vein ${ }^{(46)}$, or, if these methods fail, by manual separation. If the gloved right hand has been kept sterile throughout the later stages of labour, it may be rinsed in an antiseptic lotion to remove adherent blood and debris, coated with a film of undiluted dettol, and introduced directly into the uterus after swabbing the vulva. Strict asepsis is éssential for this operation and light general anæsthesia is desirable.

If the placenta has been expelled, the hæmorrhage will usually cease after fundal massage and expression of clot. When portions of placental tissue have been retained in utero these should be removed digitally or by the gentle use of 
a blunt flushing curette. If available, a copious intrauterine douche is recommended in all cases in which the hand has been introduced into the uterine cavity after separation of the secundines.

Stimulate the Uterus to Contract. The uterus may be stimulated to contract by kneading through the abdominal wall, by subjecting to the action of certain drugs or by the administration of a hot intrauterine douche.

Fundal massage with the bladder empty is a necessary form of stimulation in all cases of blood loss after delivery, and drugs are useful and potent supplements. Drugs used for this purpose include Caps. Ergotæ. Præp. for oral administration; Ext. Pituit. Liq., Gravitol (Bayer.), Ergometrine and other ergot extracts for intramuscular injection, and Ergometrine or well diluted Ext. Pituit. Liq. for intravenous injection. The intravenous injections are suitable for the severe cases and must be given very slowly.

The hot intrauterine douche is especially useful after manual removal of the placenta, but it is rarely available in domiciliary practice. Its use is contraindicated in cases of hæmorrhage following delivery when placenta prævia has been present, as it may wash out clot from the vessel mouths in the placental site on the lax lower uterine segment and therefore may cause further loss of blood. Prolonged use of the douche is also inadvisable when it fails to control the hæmorrhage.

Control the Hrmorrhage while the Uterus is Regaining Tone. Hendry has suggested that, by grasping the uterus per abdomen, elevating it and directing the cervix backwards towards the sacral promontory, the uterine arteries may be kinked and the hæmorrhage thereby controlled. Unfortunately this method is difficult to apply effectively when the uterus is soft and flabby, the patient is restless and intolerant of interference, and the abdominal wall is well developed, and further, it probably fails to retard the circulation in the ovarian arteries.

Aortic compression, by pressure on the aorta through the abdominal wall by the clenched fist, is difficult to apply when the patient is restless, especially if she has a muscular abdomen; it may induce shock by rough handling and forcible restraint, and it does not affect the circulation through the ovarian arteries which arise from the aorta at a high level.

Bimanual compression of the uterus between a fist in the anterior vaginal fornix and a hand on the abdomen over the posterior wall of the uterus is one of the most efficacious methods known for controlling acute uterine hæmorrhage. It should be carried out with proper aseptic precautions in all cases of severe bleeding with tardy response to simple routine treatment, especially when the hand has already been introduced into the vagina for removal of a retained placenta.

Packing the uterus is not generally favoured owing to the impossibility of observing strict asepsis and of packing efficiently in a private house, unless the circumstances are exceptional, and to the risk of converting a revealed into a concealed hæmorrhage if the uterus fails to contract upon the foreign body.

\section{Treat the Collapse ${ }^{(5)}$.}

The essentials of the treatment of the post-hæmorrhagic state are to utilize the blood remaining in the body as economically as possible, to avoid exhaustion and wasteful expenditure of energy, and to take steps to restore an efficient circulation in the shortest possible time after the hæmorrhage has been brought under control.

Warmth is provided by means of blankets and suitably protected hot water bottles, great care being taken to avoid overheating the patient and thereby causing excessive fluid loss by perspiration and further depression of the blood-pressure by dilating the skin vessels. 
The available blood may be brought into active circulation in the essential organs, especially the brain, by elevation of the foot of the bed. Bandaging of the limbs entails avoidable disturbance of the patient and is only permissible under special circumstances, and the firm application of an abdominal binder tends to hamper still further the already embarrassed respiration, and is therefore contraindicated. Cardiac stimulants are not beneficial and alcohol is harmful ${ }^{6,7)}$. Inhalation of $5 \%$ carbon dioxide in oxygen may prove helpful for stimulating the respiratory function when breathing becomes shallow.

Absolute quiet with a minimum amount of disturbance and careful avoidance of over-treatment should be insisted upon in all cases. Inessential obstetrical manipulations and transport to hospital should be postponed until the patient has recovered from the collapse. This ideal has now been made practicable in those districts in which a domiciliary emergency service ${ }^{(8,9)}$ capable of providing all the necessary equipment and personnel for dealing with any case of hæmorrhage or shock, has been organized.

Sedatives should never be withheld in the presence of uneasiness and frank restlessness ${ }^{(10)}$ and morphine salts take prime place under these circumstances. Any factors liable to aggravate the collapse, such as deprivation of fluid or food, excessive fluid loss by sweating, exposure to cold, or fatigue, must be avoided ${ }^{(11,12)}$.

Steps must also be taken to restore the fluid deficiency in the body. Glucose solution should be given by the mouth, and tap water, which is easily retained, rapidly absorbed, and gives rise to a minimum amount of discomfort may be infused into the rectum when necessary ${ }^{(13)}$.

If the blood pressure falls to $80 \mathrm{~m} . \mathrm{m}$. Hg., or fails to rise above $90 \mathrm{~m} . \mathrm{m}$. Hg. after routine nursing treatment, the infusion into a vein of a pint of ephedrineglucose-gum solution (Ephedrine Hydrochloride gr. I in a pint of $5 \%$ Glucose with $6 \%$ Gum Acacia in distilled water) or other stock colloidal mixture, is commenced immediately $(11,14,15,16,17,18,19,20,21,22,23,24,25,26,27)$. The gravity method is chosen, as delays and difficulties are less likely to be encountered when the apparatus and technique are made as simple as possible. The warm solution is introduced very slowly, at least forty minutes being allowed for the infusion of one pint of fluid to avoid overloading the heart, to prolong the beneficial effects of the procedure and to give the capillary walls time to recover, and to assist the fluid to remain in the blood vessels. If the patient becomes cyanosed or the external jugular veins become distended during infusion the fluid is entering the vein too quickly. The bed is levelled when the solution begins to flow to aid the venous return from the head and neck and to reduce the risk of cerebral œdema developing, and waterlogging of the tissues, excessive dilution of the blood cells and stagnation in the capillary reservoirs is prevented by avoidance of the introduction of fluid into the vascular system in excess of requirements. The amount infused seldom exceeds one pint ${ }^{(28)}$. As soon as the solution is passing freely into the vein, ten units of insulin are injected intramuscularly for every pint of solution containing $5 \%$ glucose to aid the adsorption and utilization of this sugar.

The restored water level is maintained and supplemented by the administration of fluid by mouth and, if necessary, by rectum, and when a cataclysm of hæmorrhage has been preceded by recurrent minor hæmorrhages, or inanition, recurrent syncopal attacks, sighing respiration or slow recovery follow the primary infusion, a blood transfusion is carried out by the indirect citrate method as soon as possible ${ }^{(29,30,31,32)}$. If a long delay is being experienced while awaiting the arrival of the donor and the patient's condition is deteriorating, the beneficial effects of the preliminary infusion may be prolonged by bandaging the limbs. 
Numerous workers are attempting to make a preparation of blood which can be stored in readiness for immediate infusion in case of emergency to simplify the technique of transfusion and to obviate delay. Preparations already used for this purpose include citrated whole blood, blood in citrated saline or glucose solution, washed erythrocyte suspension in citrate-dextrose or citrate-saline, and cadaver blood $^{(33,34,35,36,37,38,39,40,31,41,42)}$.

\section{Treatment of the Traumatic Anæmia.}

The after treatment of a patient who has suffered a considerable blood loss is most important. She should usually rest in bed for a few days longer than would have been necessary had there been no excessive hæmorrhage, and, on being allowed up and about, should live a regular life with abundant fresh air, sufficient exercise with avoidance of fatigue, and adequate sleep.

The diet should be liberal, mixed, and rich in easily digested protein ${ }^{(43)}$ with a liberal allowance of fresh fruits, salads and vegetables. Massive doses of iron $^{(44,48)}$ such as 3 to 6 I 5 grain Blaud's pill capsules daily with a vitamin A and D preparation should be taken for a period of I2 to I6 weeks. Alternative iron preparations are Ferri et Ammon. Cit. and Tab. Ferrous Sulph., of which the minimum effective daily doses should be taken as 90 and 9 grains respectively.

In occasional instances, when the anæmia is exceptionally resistant to treatment, a small blood transfusion may initiate the process of recovery and stimulate the bone marrow to increase the erythrocyte output. The prolonged course of iron therapy is still necessary in these cases, as the newly formed red cells almost invariably have a low hæmoglobin content.

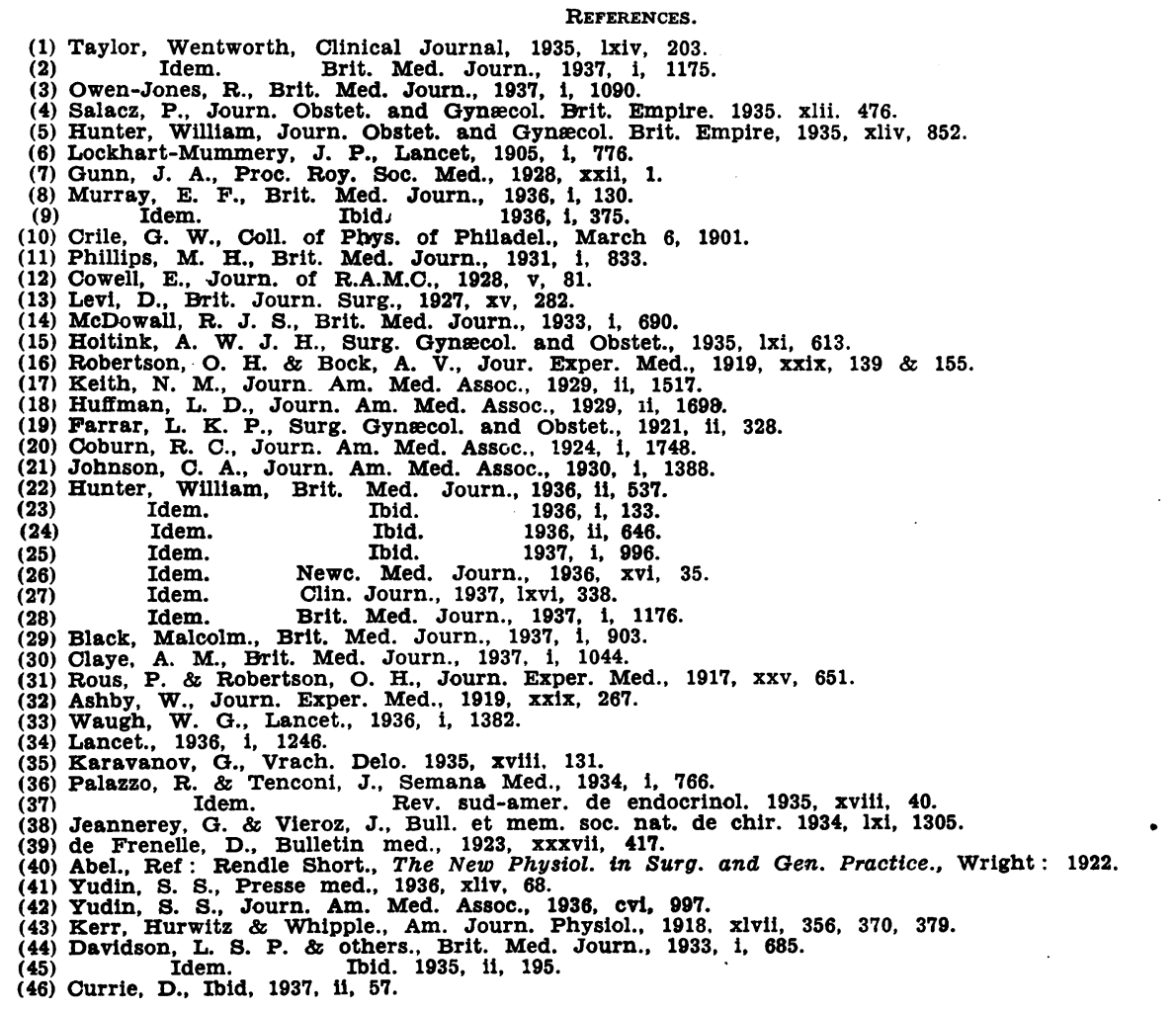

